\title{
HACIA LA GESTIÓN INTEGRADA DEL PATRIMONIO EN CLAVE TERRITORIAL: UN ANÁLISIS CRÍTICO A PARTIR DE LA EXPERIENCIA ANDALUZA ${ }^{1}$
}

\author{
Alfonso Mulero Mendigorri \\ Departamento de Geografía y Ciencias del Territorio \\ Universidad de Córdoba
}

\section{RESUMEN}

El sustancial incremento de elementos patrimoniales registrado en las tres últimas décadas ha abierto un doble debate: en torno a las razones de tal proliferación, de un lado, y sobre las estrategias y herramientas más apropiadas para gestionarlos, de otro. En tal contexto, este trabajo aborda una evaluación crítica del Sistema de Patrimonio Territorial de Andalucía (SPTA), en tanto que iniciativa destacada de alcance regional formulada con la intención de lograr una gestión integrada y coherente del prolijo patrimonio territorial reconocido en esta comunidad autónoma. El análisis general se complementa con un estudio de caso centrado en Sierra Morena (Córdoba, España).

Palabras clave: Patrimonio territorial, Plan de Ordenación del Territorio de Andalucía, Sierra Morena.

\section{ABSTRACT}

Towards an integrated management of heritage from a territorial perspective: a critical analysis based on the Andalusian experience

The substantial increase in patrimonial elements registered in the past three decades has opened a double debate: on the one hand, about the reasons for such a proliferation, and on the other, regarding the most appropriate strategies and tools to manage them. In this context, this paper addresses a critical evaluation of the Territorial Heritage System of Andalusia (SPTA), as a leading regional initiative formulated with the intention of achieving an integrated and coherent management of the wide territorial heritage of this region. The overall analysis is supplemented by a case study focused on Sierra Morena (Cordoba, Spain).

Keywords: Territorial heritage, Spatial Planning Plan of Andalusia, Sierra Morena.

\section{INTRODUCCIÓN}

En las tres últimas décadas ha tenido lugar en España una profusa intervención institucional dirigida a salvaguardar un gran número de bienes en atención a su valor e interés patrimonial. Sin duda, la consolidación del modelo autonómico y la plena incorporación del país a la Unión Europea han auspiciado la multiplicación de declaraciones protectoras tanto por iniciativa autonómica, como estatal e internacional. En términos cuantitativos la cuestión no es baladí; los espacios protegidos, en su condición de elementos destacados del comúnmente denominado patrimonio natural, abarcan ya más de la cuarta parte del territorio español -una proporción ampliamente superada en algunas autonomías (Europarc, 2012)-, y, en lo referido al patrimonio cultural, la cifra de bienes de interés cultural (BIC) registrados sobrepasa los 16.000 (Troitiño, 2011). Como es natural, tal deriva protectora y patrimonializadora viene suscitando un notable interés entre los especialistas, a la par que son muchas las razones esgrimidas para explicarla (Ortega,

Contacto: gtlmumea@uco.es

1 Este artículo ha sido realizado en el marco del proyecto de investigación financiado por el Ministerio de Ciencia e Innovación (Secretaria de Estado de Investigación), bajo el título: Dinámicas funcionales y ordenación de los espacios del "Sistema del Patrimonio Territorial" andaluz: Análisis en Andalucía occidental. (Ref. CSO2010-19278). 
1988; GDRU, 2008; Silva, 2009; Mata, 2010; Gómez, 2013). Entre ellas, no obstante, sobresalen algunas como la continua revisión y ampliación del concepto patrimonio, la mayor toma de conciencia social hacia los valores patrimoniales, la necesidad de su puesta en valor -en tanto que activos para la dinamización socioeconómica-, y, junto a todo ello, la tendencia a salvaguardar bienes y recursos territoriales ante las amenazas cada vez mayores que las nuevas dinámicas globalizadoras plantean.

En tal contexto de expansión patrimonializadora, el trabajo que ahora se presenta pretende reflexionar en torno a las dificultades existentes para alcanzar una gestión integrada de los bienes del patrimonio territorial. En primer término se presenta una evaluación crítica y de carácter teórico sobre el denominado Sistema del Patrimonio Territorial de Andalucía (SPTA), cuyas conclusiones son contrastadas, a continuación, con los resultados obtenidos de un análisis de caso centrado en la Sierra Morena cordobesa.

Metodológicamente este artículo se ha apoyado en el estudio teórico del SPTA en el contexto del Plan de Ordenación del Territorio de Andalucía, así como del conjunto de fuentes bibliográficas y documentales disponibles sobre los elementos integrantes del patrimonio natural y cultural en esta comunidad autónoma. El análisis documental se ha visto complementado por un ingente trabajo de campo consistente en la datación, localización y evaluación del conjunto de bienes del patrimonio territorial que han sido reconocidos institucionalmente en el antedicho ámbito serrano.

\section{LA PROLIFERACIÓN RECIENTE DE BIENES PATRIMONIALES Y LA TENDENCIA HACIA UNA LECTURA INTEGRADORA DE LOS MISMOS: INICIATIVAS Y DIFICULTADES}

Cifras como las citadas permiten comprender que la relación entre territorio y patrimonio se ha fortalecido de forma extraordinaria, llegando a erigirse el término patrimonio territorial en pieza clave de las nuevas fórmulas de intervención que la actual situación demanda. Se trata de una situación lógica considerando que en las últimas décadas numerosos territorios -en tanto que espacios geográficos "construidos" por el hombre (Ortega, 1988)- dotados de valores sobresalientes, reconocidos institucionalmente y apreciados socialmente, van siendo considerados ámbitos patrimoniales en sí mismos. Y, junto a ello, resulta evidente que el territorio en su conjunto es, como se ha dicho, asiento de un cada vez más extenso y heterogéneo elenco de nuevos recursos reconocidos como patrimoniales; un elenco que sólo puede ser bien interpretado en su contexto espacial y, claro está, desde miradas integradoras de una ambigua dicotomía entre patrimonio natural y patrimonio cultural que es preciso superar, aún cuando la regulación jurídico-administrativa vigente se empeñe en lo contrario.

Pues bien, en relación con este último aspecto, parece haberse establecido un amplio consenso en torno a la necesidad de interpretar y gestionar, desde una perspectiva integrada, el conjunto de bienes patrimoniales radicados en un determinado territorio. El problema, aún no resuelto adecuadamente, es cómo hacerlo, a pesar de que no faltan iniciativas encaminadas a tal fin, en la línea de las que, con clara incidencia en España, destacamos a continuación:

a) La protección de paisajes representativos. Lo más significativo al respecto es que las dos grandes instituciones internacionales en materia de preservación del patrimonio (UICN y UNESCO) han apostado por la selección y protección de paisajes concretos como estrategia para mostrar la magnífica integración entre recursos naturales y acción antrópica que tiene lugar en muchos territorios; una selección que, a nuestro juicio, es un modo de designar indirectamente paisajes de alto significado patrimonial o paisajes patrimoniales. Así, la Unión Internacional para la Conservación de la Naturaleza (UICN) incluyó, ya en 1978, la figura de Paisaje Protegido entre sus categorías protectoras de referencia, quedando definida del siguiente modo en la revisión llevada a cabo en 1992: "Área de terreno, incluyendo las costas y el mar, donde la interacción de gentes y naturaleza a lo largo del tiempo ha producido un espacio de carácter distintivo con unos valores estéticos, ecológicos y/o culturales específicos, y a menudo con una rica diversidad biológica. Salvaguardar la integridad de esta tradicional interacción es vital para la protección, el mantenimiento y la evolución del área mencionada" (Phillips, 2002). Por su parte, la UNESCO propuso la figura de Paisaje Cultural, reconocida desde 1992 en la Convención para la Protección del Patrimonio Mundial Natural y Cultural, con vistas a su aplicación sobre "obras que combinan el trabajo del hombre y la naturaleza", una definición genérica que se concreta en tres categorías específicas recogidas en la Guía Operativa para la Implementación de la Conservación del Patrimonio Mundial (Rössler, 2002).

Como es sabido, ambas iniciativas han estado presentes en la intervención administrativa española de los últimos años, bien es cierto que manteniendo su sesgo sectorial original (natural versus cultural) tanto 
en su traslación al marco normativo como en su gestión cotidiana. Así, en el ámbito de la conservación del medio natural, la legislación básica del Estado recoge, desde 1989², la figura de Paisaje Protegido, cuya definición original fue modificada por la vigente ley del Patrimonio Natural y la Biodiversidad (2007, art.34), quedando del siguiente modo: "Paisajes Protegidos son partes del territorio que las Administraciones competentes, a través del planeamiento aplicable, por sus valores naturales, estéticos y culturales, y de acuerdo con el Convenio del paisaje del Consejo de Europa, consideren merecedores de una protección especial". Junto a ello, el texto establece para esta figura varios objetivos que han de orientar su gestión allí donde se instaure: la conservación de los valores singulares que la caractericen, la preservación de la interacción armoniosa entre la naturaleza y la cultura en una zona determinada, y, en relación con lo anterior, el mantenimiento de las prácticas de carácter tradicional que contribuyan a la conservación de sus valores y recursos naturales (Mulero, 2013). Del mismo modo, la categoría de Paisaje Cultural ha suscitado un considerable interés en el marco de las administraciones competentes. A escala estatal el Ministerio de Educación, Cultura y Deportes ha impulsado un "Plan Nacional de Paisaje Cultural" aprobado recientemente (2012), y, por otra parte, es evidente la difusión de esta figura en el contexto autonómico: las leyes de patrimonio histórico y cultural de Cantabria (1998), La Rioja (2004) y Navarra (2005) la han incorporado y en todas ellas hace referencia a un espacio representativo de la interacción entre el trabajo del hombre y la naturaleza; en Andalucía, los trabajos de análisis, inventario y catalogación de paisajes culturales que lleva a cabo el Instituto Andaluz de Patrimonio Histórico (Fernández, 2010; Rodrigo, et al., 2012), son otra buena muestra de ello.

b) La delimitación y salvaguarda de territorios específicos caracterizados por el valor y la diversidad de sus elementos patrimoniales. Una estrategia que se ha concretado, por ejemplo, en la creación de la figura de Zona Patrimonial, contemplada en la Ley del Patrimonio Histórico de Andalucía (2007, art. 26.8)) con la siguiente definición: " (...) aquellos territorios o espacios que constituyen un conjunto patrimonial, diverso y complementario, integrado por bienes diacrónicos representativos de la evolución humana, que poseen un valor de uso y disfrute para la colectividad y, en su caso, valores paisajísticos y ambientales". Recuérdese que áreas muy significativas desde el punto de vista patrimonial, como la Vega de Granada o la Cuenca Minera de Riotinto (Huelva), entre otros varios, cuentan ya con la declaración de Zona Patrimonial.

c) El diseño de herramientas específicas que permitan una gestión integrada del patrimonio territorial en ámbitos concretos. Se trata de un planteamiento en consonancia con la Estrategia Territorial Europea, que desde 1999 aboga por un tratamiento integrado del patrimonio en el diseño del modelo territorial de la Unión Europea, a la vez que insiste en la necesidad de convergencia de las actuaciones de conservación y puesta en valor del patrimonio cultural y natural, en pos de un uso prudente de los recursos y de un territorio de calidad (Mata, 2010). Sin duda es la apuesta más ambiciosa y prometedora entre las enunciadas, aunque, a nuestro juicio, su viabilidad pasa en la práctica por la resolución de, al menos, cuatro retos básicos:

$1^{\circ}$ Es preciso lograr una definición más ajustada y mejor fundamentada de los elementos que integran el "patrimonio territorial", cuestión ésta en modo alguno baladí. De hecho, ya se encuentra muy extendido el criterio de considerar junto a los bienes de carácter oficial -los seleccionados y protegidos por la legislación competente- otros muchos cuya dimensión patrimonial descansa en valores muy diversos, aunque siempre bajo la condición de un sólido e incuestionable reconocimiento social (Ortega, 1998; Castillo, 2007; Feria, 2010).

Por otra parte, es sabido que los bienes culturales y naturales que hoy integran los catálogos oficiales se han ido seleccionando conforme a criterios dispares y coyunturales, sin que, en la mayoría de los casos, se haya producido la necesaria revisión y actualización de los mismos; ésta es una realidad que plantea graves problemas de coherencia a cualquier iniciativa sólida de gestión integrada del patrimonio territorial. En consecuencia, parece prioritario lograr un consenso claro entre lo que debe y no debe considerarse elemento integrante del patrimonio territorial.

$2^{\circ}$ En segundo término, es preciso estipular cómo gestionar, con criterios de integración, un patrimonio territorial de la magnitud y diversidad del considerado, en un contexto jurídico determinado por la existencia de leyes sectoriales reguladoras de lo natural y de lo cultural, que además han sido confeccionadas normalmente de espaldas las unas a las otras y sin apenas puntos de encuentro. Las leyes marco hoy vigentes (Ley de Patrimonio Histórico Español de 1985 y Ley del Patrimonio

2 Ley 4/1989, de 27 de marzo, de Conservación de Espacios Naturales y de la Flora y Fauna Silvestres. 
Natural y de la Biodiversidad de 2007) no son sino el principal exponente de una situación que es la habitual en los instrumentos normativos autonómicos.

$3^{\circ}$ El discurso en torno a la necesidad de una gestión integrada del patrimonio territorial debe igualmente clarificarse. Por ejemplo, se ha llegado a afirmar que sólo a través de la gestión conjunta de los activos patrimoniales con que cuenta un territorio se podrá conseguir que estos se conviertan en un recurso para su desarrollo (GDRU, 2008); sin embargo, se trata de una aseveración discutible, con la que no podemos estar de acuerdo. En realidad, la dinamización socioeconómica de un territorio, cuando ha tenido que llegar, lo ha hecho sin el requisito previo de un manejo integrado de su patrimonio; por citar un caso, considérense los logros alcanzados con la simple declaración de espacios naturales protegidos y la repercusión en sus áreas de influencia. Parece que la aspiración hacia una gestión armónica de los activos del patrimonio territorial ha de apoyarse, más que en razones vinculadas a la coyuntura económica, en otras de índole estructural y orientadas a alcanzar un uso prudente de los recursos y, a la postre, un territorio de calidad.

$4^{\circ} \mathrm{Y}$, finalmente, la cuestión de la escala. ¿Qué escala y ámbito de actuación son los propicios para aplicar una ordenación y gestión integradas del patrimonio territorial? En algún trabajo reciente se ha defendido la idoneidad de las escalas intermedias -que se concretan en un rango situado entre un mínimo de varios centenares de kilómetros cuadrados y un máximo de 10.000- o "aquéllas en que la confluencia del medio y la acción antrópica ha conseguido modelar territorios relativamente individualizables y singulares (...)" (Feria, 2010: 134). En cualquier caso, éste es otro de los asuntos cruciales pendientes de resolución y de consenso.

Pues bien, a pesar de la complejidad de esta tercera vía y de los considerables retos que plantea su puesta en práctica, en la comunidad autónoma andaluza se ha impulsado una herramienta específica o sistema para la gestión integrada del patrimonio territorial, a través del Plan de Ordenación del Territorio de Andalucía (en adelante POTA), vigente desde 2006. Su alcance y significado justifican un tratamiento algo más detallado en las siguientes páginas.

\section{EL PLAN DE ORDENACIÓN DEL TERRITORIO DE ANDALUCÍA (POTA) Y LA APUESTA POR UNA INTEGRACIÓN DEL PATRIMONIO EN CLAVE TERRITORIAL}

El documento marco de la planificación territorial andaluza desarrolla una de las principales iniciativas administrativas encaminadas a la resolución de la interrogante arriba enunciada: ¿cómo lograr una gestión integrada de los bienes patrimoniales en clave territorial? o ¿cómo integrar coherentemente patrimonio natural, patrimonio cultural y paisaje? Para algunos especialistas, el camino correcto exige que "el sistema patrimonial aparezca incardinado en los programas de desarrollo territorial, considerando el manejo de los recursos naturales, culturales y paisajísticos como una oportunidad que ofrecer a la sociedad" (Troitiño, 2011: 567). Y en esta línea, ya en los años noventa, se posicionaron los redactores del avance del POTA al diseñar el denominado Sistema de Patrimonio Territorial de Andalucía (en adelante SPTA), una iniciativa sin precedentes a esta escala que ha llegado a ser valorada como ejemplo de buena práctica en un espacio donde la complementariedad y sinergia de valores culturales y naturales en el territorio es muy elevada (Mata, 2010). El SPTA figura entre las diversas estrategias de desarrollo territorial contempladas en el POTA, y tiene encomendada la misión de procurar una "gestión y ordenación unitaria" de los principales elementos que conforman el patrimonio natural y cultural de la región (Zoido, 2011). Se pretende, en definitiva, superar un manejo apoyado en planteamientos excesivamente parciales, desde un "tradicional entendimiento de los bienes patrimoniales como elementos aislados, hitos sin conexión con el territorio y con el resto de bienes patrimoniales" (POTA, 2006: 131).

En su diseño teórico, el SPTA se apoya en tres pilares esenciales. El primero de ellos es una base espacial, resultado de la división de la comunidad autónoma en 33 Unidades Territoriales (figura 1), desde el presupuesto de que tales ámbitos comparten unos componentes ecológicos, históricos y culturales comunes, es decir, un marco identitario que puede facilitar la planificación y gestión unitarias de sus respectivos bienes patrimoniales. El segundo es el Programa Coordinado con que contará cada Unidad Territorial, o marco de consenso entre las diferentes instancias administrativas del gobierno regional, a fin de armonizar las políticas de protección patrimonial vigentes (naturales, culturales, paisajísticas) con el resto de las grandes herramientas de la ordenación territorial. Por último, es clave el Sistema de Información de los espacios y bienes catalogados del patrimonio territorial, concebido como plataforma compartida por varias instancias de la Junta de Andalucía (Consejerías de Obras Públicas, Medio Ambiente 
y Cultura), en colaboración con los restantes organismos competentes en la materia; obviamente esta herramienta resulta imprescindible con vistas a la gestión de un ingente número de bienes patrimoniales, por lo general poco imbricados, regulados por normativas muy heterogéneas y recogidos en catálogos e inventarios de la más variada índole.

Figura 1. Unidades territoriales definidas por el Plan de Ordenación de Ordenación del Territorio de Andalucía.

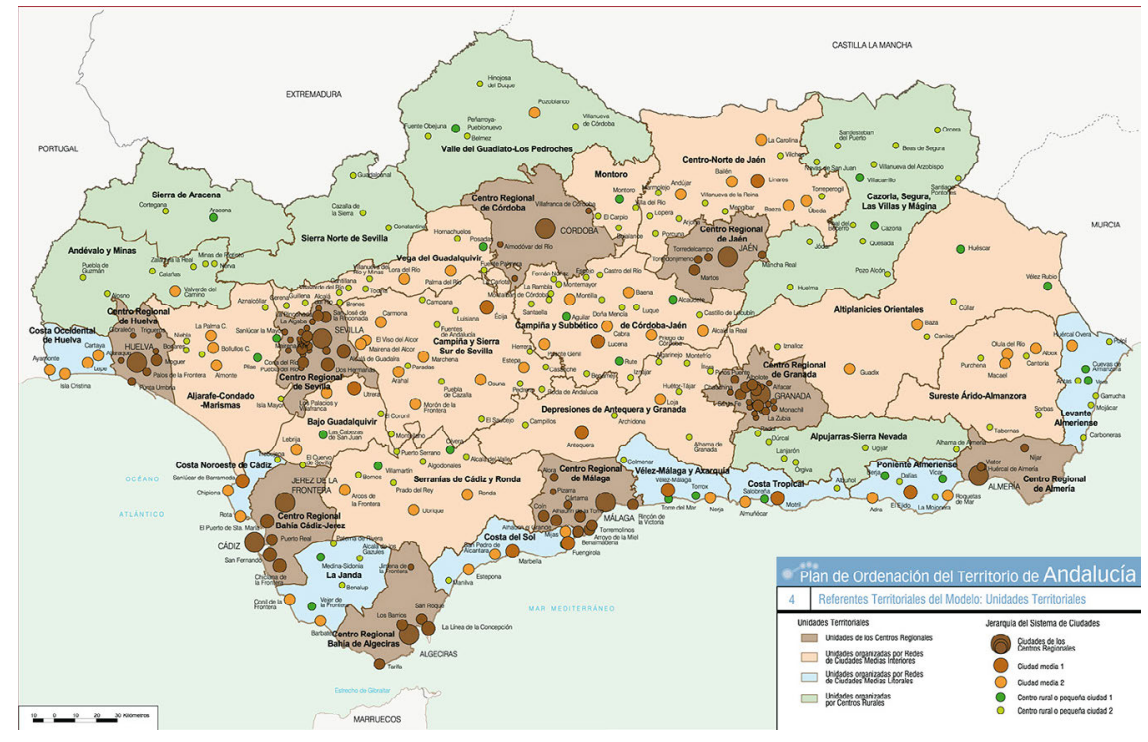

Fuente: Plan de Ordenación del Territorio de Andalucía, 2006.

Figura 2. Principales elementos del Sistema de Patrimonio Territorial de Andalucía.

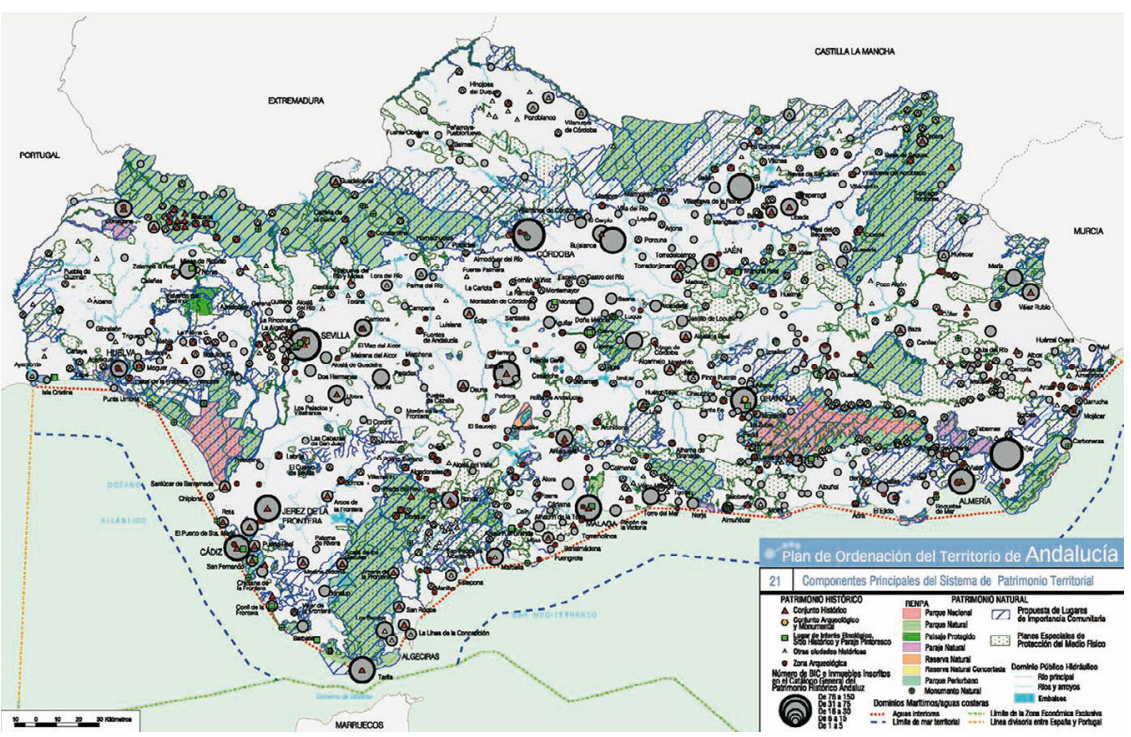

Fuente: Plan de Ordenación del Territorio de Andalucía, 2006.

En definitiva, a tenor de los anteriores planteamientos, podemos coincidir con Mata (2010) en su valoración del SPTA como iniciativa ejemplar, pero sólo en un sentido puramente propositivo o teórico, pues, a nuestro juicio, en la práctica adolece de notables carencias e interrogantes que cuestionan seriamente su viabilidad (Mulero et al, 2011). De entrada, las Unidades Territoriales han sido delimitadas en orden a criterios dominantes de centralidad urbana, y plantean serios inconvenientes e incongruencias: entre ellas, la decisión de dividir términos municipales o grandes parques naturales entre dos unidades territoriales limítrofes. Se olvida, por ejemplo, que éstos últimos son la pieza clave del patrimonio natural andaluz y que se encuentran sujetos a instrumentos de planificación de considerable alcance que requie- 
ren unidad de gestión (Planes de Ordenación de los Recursos Naturales, Planes Rectores de Uso y Gestión, Planes de Desarrollo Sostenible) (compárense las figuras 1 y 2).

Por otra parte, el repertorio de los espacios y bienes naturales y culturales que han de integrar inicialmente el SPTA no sólo es impreciso, complejo y excesivamente prolijo (tabla 1), sino que progresivamente habrá de recoger todos aquellos nuevos espacios y elementos que se incorporen a algunos de los catálogos de protección vigentes, así como los resultantes de los estudios territoriales y sectoriales que contengan inventarios de bienes del patrimonio, susceptibles de ser catalogados por las diferentes administraciones públicas (inventario de paisajes, inventario del patrimonio industrial y minero, etc.) (tabla 2). No es de extrañar, por tanto, que el referido Sistema de Información no haya logrado desarrollarse hasta la fecha.

Finalmente, en el modelo de gestión propuesto para el SPTA subyacen, en nuestra opinión, dos problemas esenciales: de un lado, no se han concretado las Estrategias de planificación y gestión integradas del Patrimonio Territorial -que el POTA contempla como pieza clave de su planteamiento-, y, de otro, resulta preocupante la desconexión real existente, a efectos patrimoniales, entre los diversos instrumentos de planificación; nótese al respecto que muchos de los planes de ordenación y gestión de los espacios protegidos recientemente aprobados en Andalucía (PORN y PRUG) -que inciden sobre una parte sustancial de la región-, no han incorporado los nuevos presupuestos hacia los que debería encaminarse la gestión del patrimonio territorial, o, en la misma línea, recuérdese que los ámbitos de actuación delimitados para muchos de los Planes Subregionales de Ordenación del Territorio ya aprobados -Aglomeración urbana de Sevilla, Sur de Córdoba, Sierra de Segura, etc.- difieren sustancialmente de las Unidades Territoriales establecidas en el POTA, lo que plantea graves interrogantes acerca del engarce que podrán tener en el futuro ambas circunscripciones territoriales.

Tabla 1. Recursos que integran inicialmente el Sistema de Patrimonio Territorial de Andalucía.

\begin{tabular}{|c|}
\hline $\begin{array}{l}\text { a) Los espacios naturales protegidos y bienes culturales protegidos por figuras internacionales (Reservas de la Biosfera, Patrimonio de } \\
\text { la Humanidad, Humedales del Convenio RAMSAR, Zonas Especialmente Protegidas de Importancia para el Mediterráneo), así como } \\
\text { los Lugares de Importancia Comunitaria de la región biogeográfica mediterránea incluidos en la Decisión de la Comisión, de } 19 \text { de } \\
\text { julio de 2006, por la que se adopta, de conformidad con la Directiva 92/43/CEE del Consejo, la lista de los citados lugares. }\end{array}$ \\
\hline b) La Red de Espacios Naturales Protegidos de Andalucía. \\
\hline $\begin{array}{l}\text { c) Los espacios incluidos en el Catálogo de Espacios y Bienes Protegidos de los Planes Especiales de Protección del Medio Físico de ám- } \\
\text { bito provincial o, en su caso, los suelos no urbanizable calificados de especial protección por el planeamiento territorial o urbanístico. }\end{array}$ \\
\hline d) Las zonas que constituyen el dominio público hidráulico y marítimo terrestre \\
\hline e) Las vías pecuarias y otros «caminos naturales». \\
\hline f) Los inventarios de georrecursos y de humedales. \\
\hline g) Ciudades históricas protegidas como Conjuntos Históricos \\
\hline h) Otras Ciudades históricas no protegidas pero equivalentes en valores a los Conjuntos Históricos. \\
\hline i) Patrimonio inmueble con categoría de BIC o inscrito en el Catálogo General del Patrimonio Histórico de Andalucía \\
\hline j) Otros yacimientos arqueológicos y elementos del patrimonio cultural (hábitats de cuevas, arquitectura popular, etc.). \\
\hline
\end{tabular}

Fuente: Plan de Ordenación del Territorio de Andalucía, 2006. Elaboración propia.

Tabla 2. Otros elementos que, junto a los reconocidos en el SPTA, habrán de integrar el Sistema de Información de espacios y bienes catalogados del Patrimonio Territorial.

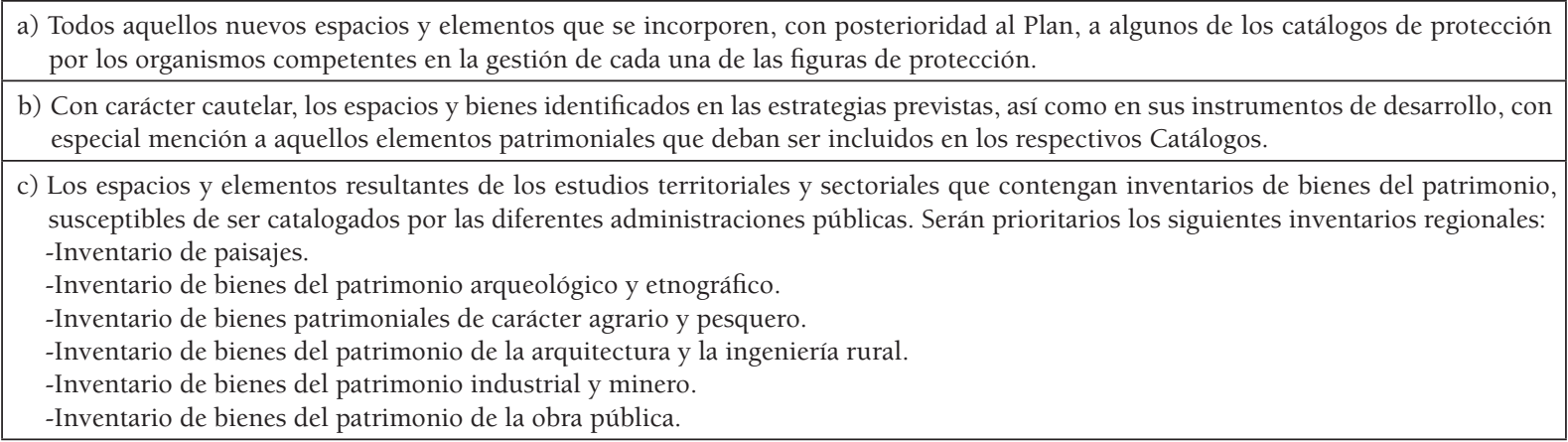




\section{EL SISTEMA DE PATRIMONIO TERRITORIAL DE ANDALUCÍA Y SU APLICABILIDAD EN ÁMBITOS CONCRETOS: ESTUDIO DE CASO EN LA SIERRA MORENA CORDOBESA}

Junto con los anteriores juicios de carácter general, se hace necesaria una evaluación de la viabilidad del SPTA en territorios concretos, al objeto de contrastar mejor su idoneidad como herramienta de referencia. Con tal finalidad se ha llevado a cabo un estudio en el norte de la provincia de Córdoba, orientado a valorar la situación de los elementos patrimoniales allí radicados y las posibilidades de aplicación de las directrices marcadas por el POTA. Entre los resultados obtenidos, nos limitaremos ahora a señalar la existencia de tres grandes obstáculos que, a nuestro juicio, testimonian las limitaciones generales de la propuesta andaluza -arriba citadas-, a la vez que cuestionan seriamente su viabilidad práctica en relación con el patrimonio territorial.

\subsection{El primer escollo: la delimitación del ámbito sobre el que aplicar una gestión unitaria del patrimonio territorial}

Como se ha indicado más arriba, el Sistema de Patrimonio Territorial de Andalucía se asienta sobre una base de 33 Unidades Territoriales que pretenden ser coherentes y operativas, por cuanto, se afirma, en su delimitación han sido tenidos en cuenta rasgos esenciales de tipo ecológico, histórico y cultural. La Sierra Morena cordobesa ha quedado englobada en su mayor parte en la unidad Valle del Guadiato-Los Pedroches (figura 3 y tabla 3), a través de la unión de dos ámbitos serranos bien diferenciados en la mayor parte de los ejercicios de comarcalización efectuados hasta la fecha, ya sea con criterios administrativos, técnicos o científicos. De este modo el resultado es un territorio equivalente al $7.2 \%$ de la región, con una notable diversidad administrativa (26 términos municipales de muy distinta entidad), pero inconsistente desde un punto de vista geográfico, a tenor de su clara discontinuidad físico-natural. En principio, pudiera parecer que el criterio dominante en la delimitación de esta Unidad Territorial habría sido su identificación con la Sierra Morena cordobesa; sin embargo, la simple observación de las figuras adjuntas permite comprobar que tal correspondencia no existe, y que una parte sustancial de la misma ha quedado adscrita a otras Unidades Territoriales limítrofes: es lo que ha ocurrido con diversos municipios eminentemente serranos (Villaviciosa de Córdoba, Adamuz y Obejo), y también con otros de carácter mixto Sierra-Valle (la capital provincial o Montoro). Como resultado, asistimos a situaciones incomprensibles como la inclusión de la extensa y quebrada Sierra de Hornachuelos, frente a la exclusión de las serranías limítrofes de Villaviciosa de Córdoba o Posadas, rompiendo la continuidad ecológica en aras de criterios poco o nada explicitados.

Figura 3. Mapa de la Unidad Territorial Los Pedroches-Valle del Guadiato

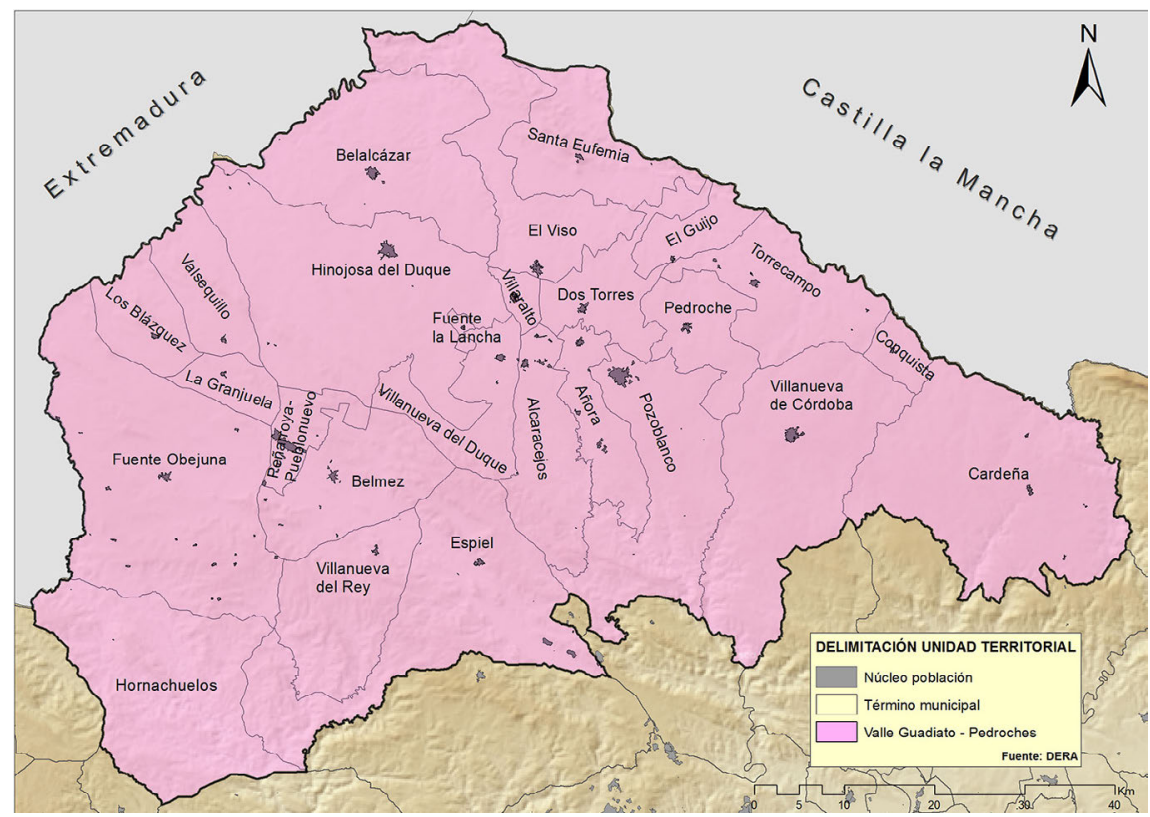

Fuente: Plan de Ordenación del Territorio de Andalucía, 2006. Elaboración propia 
Tabla 3. Municipios que integran la Unidad Territorial Los Pedroches-Valle del Guadiato

\begin{tabular}{|l|c|c|}
\hline \multicolumn{1}{|c|}{ Municipio } & $\begin{array}{c}\text { Extensión } \\
\left(\mathbf{k m}^{2}\right)\end{array}$ & $\begin{array}{c}\text { Población } \\
(\mathbf{2 0 1 2})\end{array}$ \\
\hline Alcaracejos & 175.6 & 1.545 \\
\hline Añora & 112.6 & 1.556 \\
\hline Belalcázar & 356 & 3.486 \\
\hline Belmez & 207.4 & 3.162 \\
\hline Blázquez, Los & 102.7 & 727 \\
\hline Cardeña & 512.9 & 1.669 \\
\hline Conquista & 38.5 & 461 \\
\hline Dos Torres & 129.1 & 2.524 \\
\hline Espiel & 437.3 & 2.464 \\
\hline Fuente la Lancha & 7.8 & 369 \\
\hline Fuente Obejuna & 591.4 & 5.129 \\
\hline Granjuela (La) & 56.1 & 510 \\
\hline Guijo, (El) & 67.3 & 411 \\
\hline Hinojosa del Duque & 531.5 & 7.250 \\
\hline Hornachuelos * & 909.2 & 4.694 \\
\hline Pedroche & 121.6 & 1.633 \\
\hline Peñarroya-Pueblonuevo & 64.9 & 11.651 \\
\hline Pozoblanco & 329.9 & 17.683 \\
\hline Santa Eufemia & 187.3 & 921 \\
\hline Torrecampo & 196.5 & 1.249 \\
\hline Valsequillo & 121.8 & 399 \\
\hline Villanueva de Córdoba & 429.5 & 9.440 \\
\hline Villanueva del Duque & 137.6 & 1.611 \\
\hline Villanueva del Rey & 215.8 & 1.167 \\
\hline Villaralto & 24.1 & 1.282 \\
\hline Viso (El) & 254.4 & 2.763 \\
\hline Total & 6.318 .8 & 85.756 \\
\hline
\end{tabular}

* Se han incluido los datos del municipio en su conjunto, aunque sólo su sector serrano forma parte de esta Unidad Territorial.

Fuente: INE. Elaboración propia.

\subsection{El segundo escollo: la estipulación de los elementos que conforman el patrimonio territorial}

Como ya se ha dicho, una de las grandes limitaciones estructurales que contiene la propuesta enunciada en el POTA es justamente la imprecisión y complejidad del catálogo de los espacios y bienes -naturales y culturales- que han de integrar el Sistema de Patrimonio Territorial de Andalucía. Esta es una cuestión capital y, como tal, ha sido minuciosamente analizada y ponderada por Florido (2013) en un trabajo que viene a demostrar de forma contundente la extrema dificultad, cuando no la imposibilidad, de articular un sistema coherente de gestión del patrimonio territorial atendiendo a lo estipulado en el documento marco.

A nuestro juicio, el problema de fondo se encuentra en la formulación teórica del Plan, cuyo afán de exhaustividad a la hora de enumerar los elementos que han de integrar el patrimonio territorial no tiene correspondencia con su concreción e identificación en la práctica, dando lugar a una notable confusión ${ }^{3}$.

3 Un problema añadido es la falta de convergencia entre lo estipulado por el POTA y lo regulado en la normativa estatal. Por ejemplo, en lo relativo a los componentes que integran el Patrimonio Natural, la encargada de su regulación es la Ley estatal 42/2007, de 13 de diciembre, del Patrimonio Natural y de la Biodiversidad. Ésta, a la hora de concretar en la práctica qué bienes de un territorio integran lo esencial de su patrimonio natural, establece un elenco también prolijo pero sustancialmente diferente al estipulado en el Plan de Ordenación del Territorio de Andalucía. 
En determinados casos, ésta es debida a la falta de especificación de algunos de los elementos indicados (sería el caso de los consignados como "Caminos Naturales", "Ciudades históricas no protegidas pero equivalentes en valores a los Conjuntos Históricos", y "Otros yacimientos arqueológicos y elementos del patrimonio cultural"). En otras ocasiones la dificultad se sitúa en la inexistencia de catalogación y delimitación efectiva de los mismos ("Vías Pecuarias", "Zonas que constituyen el dominio público hidráulico terrestre", "Georrecursos y Humedales", etc.). También son claros los problemas vinculados a la indefinición y falta de regulación jurídica (por ejemplo, al considerar el complejo elenco de espacios naturales protegidos por instrumentos nacionales e internacionales que se superponen), o a la modificación periódica que sufren determinados bienes catalogados (Catálogos de los Planes Especiales de Protección del Medio Físico; Suelos no urbanizables calificados de especial protección por el planeamiento). Además, al no haberse desarrollado el instrumento clave al que el POTA encomienda esta finalidad (Sistema de Información de Espacios y Bienes Catalogados del Patrimonio Territorial), la ausencia de directrices para la clarificación de los problemas apuntados es total.

Por lo tanto, puede convenirse que dos cuestiones esenciales están necesitadas de revisión: la propia inconcreción conceptual en torno a lo que debe considerarse patrimonio territorial, y, desde esta carencia, la apuesta de los redactores del POTA por un modelo exhaustivo que considera patrimonio territorial no sólo a todo bien y espacio que ha sido protegido y gestionado en cuanto tal, sino también a todo aquel de propiedad o dominio público, o que haya sido inventariado o catalogados con alguna finalidad informativa o preventiva, o que pueda serlo en un futuro. Este planteamiento impone una noción de patrimonio territorial tan extraordinariamente amplia y compleja, y con tantos instrumentos normativos y administrativos en juego, que aspirar a una gestión integrada del mismo es poco menos que una quimera. Ni siquiera parece viable lograr su conocimiento preciso en cada una de las Unidades Territoriales delimitadas por el POTA, y prueba de ello es que nada se ha hecho en relación con los sistemas de información previstos para tal fin, según se ha comentado con anterioridad. Entonces ¿cómo avanzar en la concreción del patrimonio territorial de un ámbito cualquiera?

\subsubsection{El reconocimiento institucional como criterio inicial para el establecimiento de los elementos esenciales del patrimonio territorial}

A tenor de lo dicho, parece claro que no todos los elementos seleccionados como integrantes del SPTA pueden ni deben tener la misma importancia a la hora de articular el sistema patrimonial de cada Unidad Territorial. Ante la imposibilidad de catalogación efectiva, de delimitación precisa, y de regulación jurídica sólida de muchos de ellos, el sentido común aconseja otorgar prevalencia a los bienes patrimoniales que han sido objeto de una delimitación y declaración específica con la finalidad de dotarlos de una protección efectiva y diferenciada; es decir, aquéllos dotados de un sólido respaldo institucional y de un reconocimiento y regulación en la legislación básica. En este sentido, aun coincidiendo con Prats (2004) en que en última instancia todo proceso de patrimonialización es siempre un acto de poder, el conjunto de la intervención político-normativa presenta demasiados y desiguales frentes, de modo que es necesario seleccionar. Así, creemos aconsejable distinguir entre un patrimonio territorial institucionalizado y regulado -atendiendo a lo que la propia legislación estatal y autonómica reconoce en sus textos básicos ${ }^{4}$, y aquel otro que sólo ha sido catalogado o inventariado administrativamente, de muy diversas formas y con criterios dispares según la comunidad autónoma en que nos situemos.

Desde la anterior postura, parece claro que el referente de los bienes integrantes del patrimonio natural han de ser los espacios que integran la RENPA o Red de Espacios Naturales Protegidos de Andalucía, pues ésta abarca tanto a los espacios que han sido protegidos conforme a la normativa autonómica y estatal, como a aquellos otros amparados y gestionados por instrumentos internacionales diversos. Del mismo modo, en lo relativo al patrimonio cultural, tal posición preeminente habrán de ocuparla los elementos inscritos en el Catálogo General de Patrimonio Histórico (Bienes de Interés Cultural y Bienes de Catalogación General), cuya cifra, próxima a los 4.000 registros, es ya de por sí muy cuantiosa. El resto de los elementos consignados en el elenco propuesto en el POTA o no gozan de protección o la que tienen es en general de carácter preventivo o propositivo, y raramente disponen de instrumentos específicos para

\footnotetext{
4 Ley 42/2007, de 13 de diciembre, del Patrimonio Natural y de la Biodiversidad; Ley 16/1985, de 25 junio, del Patrimonio Histórico Español; Ley 14/2007, de 26 de noviembre, del Patrimonio Histórico de Andalucía; Ley 2/1989, de 18 de julio, por la que se crea el Inventario de Espacios Naturales Protegidos de Andalucía y se establecen medidas adicionales para su protección.
} 
su manejo y gestión: en esta situación han de encuadrarse, por ejemplo, las alrededor de 20.000 piezas registradas en el Inventario de Bienes Reconocidos del Patrimonio Cultural de Andalucía, o, en términos de patrimonio natural, el dominio público hidráulico, los humedales, las vías pecuarias y tantos otros elementos de similar perfil. Incluso cabría incluir en este grupo a los espacios catalogados por los Planes Especiales de Protección del Medio Físico a mediados de los pasados años ochenta, pues además de carecer de una gestión específica en tanto tales, su existencia es sumamente inestable al estar sujeta a lo que sobre ellos dispongan la planificación urbanística y la de ordenación territorial de ámbito subregional5; de hecho, algunos de los espacios originalmente recogidos en los respectivos catálogos provinciales ya han visto anulada o modificada su condición inicial.

\subsubsection{Alcance y significado del Patrimonio Territorial institucionalizado en Los Pedroches-Valle del Guadiato}

A tenor de los criterios antedichos, en la tabla 4 y la figura 4 se muestra la situación del patrimonio territorial de mayor importancia y significado en el norte de la provincia de Córdoba. Su observación, en comparación con el conjunto de la Sierra Morena andaluza, permite concluir inmediatamente que la intervención institucional llevada a cabo en aquel ámbito ha sido francamente exigua, tal como reflejan incluso los mapas a pequeña escala (figura 2). Se trata, en definitiva, de una situación singular ya destacada en un trabajo pionero sobre el patrimonio territorial andaluz, cuyos autores situaron nuestro ámbito de análisis entre aquéllos "(...) con escasos recursos naturales y culturales reconocidos, no tanto por su inexistencia como por una valoración diferencial o por la aplicación de criterios de protección que no se adecúan bien a sus características. En estas circunstancias aparece buena parte de la provincia de Granada, la Sierra de la Contraviesa, el Alto Almanzora y, sobre todo, casi la totalidad de las comarcas de los Pedroches y del Andévalo, en este caso con la excepción de su banda occidental" (GDRU, 2008, 309). Una valoración muy acertada sin duda, por cuanto, en buena medida, la situación patrimonial del sector analizado viene obedeciendo a factores coyunturales, en la línea de los siguientes:

- Los criterios seguidos en la delimitación y declaración de los seis parques naturales -estandarte del patrimonio natural en el conjunto de Sierra Morena-, fueron dispares e incluso opuestos de unas a otras provincias. Así, en la provincia de Córdoba se impusieron criterios técnicos más restrictivos, dando lugar a parques más reducidos y de orientación esencialmente conservacionista (Sierra de Hornachuelos; Sierra de Cardeña y Montoro), mientras que en otros casos se optó por un modelo de gran parque, con numerosos núcleos urbanos en su interior y un perfil más "desarrollista" (Sierra de Aracena y Picos de Aroche; Sierra Norte de Sevilla) (Mulero, 2001).

- La existencia de una importante actividad agroganadera en tiempos recientes y aún presentes, ha modelado y consolidado en el norte cordobés unos paisajes agrarios en muchos sentidos excepcionales (muy reconocidos y valorados en algún caso, como la dehesa), pero no "idóneos" conforme a los criterios de patrimonialización imperantes en las grandes iniciativas institucionales auspiciadas desde los pasados años ochenta. No obstante, algunas nuevas fórmulas (Paisajes Culturales, Zonas Patrimoniales...) parecen respaldar criterios diferentes que, a medio plazo, podrían dar lugar a modificaciones sustanciales del mapa patrimonial actual.

- A nuestro juicio, algunos de los elementos reconocidos como Bienes de Interés Cultural y Bienes de Catalogación General (Cuadro 4), han sido seleccionados en función de impulsos coyunturales, políticos y/o técnicos, lo que explica la proliferación de designaciones en algunas fechas concretas, las acusadas diferencias intermunicipales que se registran, y también algunas ausencias notables. Además, en términos cuantitativos, resulta evidente la escasa atención prestada al territorio que nos ocupa, cuyos 39 BIC contrastan con los casi cuatro mil bienes de esta naturaleza que integran el catálogo regional.

- Por último, es preciso insistir en que, en la Sierra Morena cordobesa, las políticas "estancas" de designación de bienes patrimoniales han logrado como resultado principal un doble catálogo, de elementos naturales y culturales, sin ningún tipo de vinculación entre sí, ni de regulación común en los principales instrumentos de planificación con incidencia en el área, como se tratará en el siguiente apartado.

5 En efecto, algunos espacios integrantes de los catálogos de bienes y espacios protegidos incorporados en los Planes Especiales de Protección del Medio Físico, han visto ya alterada su condición: bien por una modificación introducida en la revisión del planeamiento urbanístico, bien por la aprobación de un Plan de Ordenación del Territorio de ámbito subregional, que deja sin efecto lo catalogado por el respectivo PEPMF provincial, tal y como ha ocurrido recientemente con el POTAS del Sur de Córdoba. 
Tabla 4. Elementos institucionalizados del patrimonio territorial (con tutela y gestión específicas) en la Unidad Territorial Pedroches-Valle del Guadiato

Patrimonio de dominante natural

\begin{tabular}{|c|c|c|c|}
\hline $\begin{array}{l}\mathrm{N}^{\circ} \text { de orden } \\
\text { en mapa }\end{array}$ & Elemento patrimonial & $\begin{array}{l}\text { Reconocimiento institucional } \\
\text { y grado de protección }\end{array}$ & Extensión (ha) \\
\hline 1 & Sierra de Hornachuelos (1) & $\begin{array}{l}\text { Parque Natural } \\
\text { ZEC y ZEPA (ES0000050) } \\
\text { Reserva de la Biosfera (Dehesas de Sierra Morena) }\end{array}$ & 60.032 \\
\hline 2 & Sierra de Cardeña y Montoro (1) & $\begin{array}{l}\text { Parque Natural } \\
\text { ZEC y ZEPA (ES6130001) }\end{array}$ & 38.449 \\
\hline 3 & Fuente La Zarza & Parque Periurbano & 315 \\
\hline 4 & Sierra de Santa Eufemia & LIC (ES6130003) & $10.651,55$ \\
\hline 5 & Río Guadalmez & LIC (ES6130004) & $10.586,89$ \\
\hline 6 & $\begin{array}{l}\text { Suroeste de la Sierra de Cardeña y } \\
\text { Montoro (1) }\end{array}$ & LIC (ES6130005) & $33.070,94$ \\
\hline 7 & Guadalmellato (1) & LIC (ES6130006) & $39.795,54$ \\
\hline 8 & Ríos Cuzna y Gato & LIC (ES6130009) & 112,09 \\
\hline 9 & Río Guadamatilla y Arroyo del Tamujar & LIC (ES6130010) & 135,94 \\
\hline 10 & Río Guadamatilla & LIC (ES6130011) & 13,73 \\
\hline 11 & Río Zújar & LIC (ES6130012) & 108,50 \\
\hline 12 & Arroyo de Ventas Nuevas & LIC (ES6130014) & 4,60 \\
\hline 13 & Río Guadalbarbo & LIC (ES6130016) & 9,43 \\
\hline 14 & Alto Guadiato & ZEPA (ES6130017) & $33.930,72$ \\
\hline 15 & Guadiato-Bembézar (1) & LIC (ES613007) & $114.345,41$ \\
\hline
\end{tabular}

(1) Espacios con presencia parcial en la Unidad Territorial

LIC: Lugar de Interés Comunitario (Red Natura 2000); ZEC: Zona Especial de Conservación (Red Natura 2000);

ZEPA: Zona de Especial Protección para las Aves.

Patrimonio de dominante cultural

\begin{tabular}{|l|l|l|}
\hline \multicolumn{1}{|c|}{ Elemento patrimonial } & \multicolumn{1}{|c|}{$\begin{array}{c}\text { Reconocimiento institucional } \\
\text { y grado de protección }\end{array}$} & \multicolumn{1}{|c|}{ Término municipal } \\
\hline Pósito & CG & Añora \\
\hline Ermita de Ntra. Sra. de Gracia de las Alcantarillas & CG & Belalcázar \\
\hline $\begin{array}{l}\text { Antiguo Convento de San Francisco de los Santos Mártires de } \\
\text { Marruecos }\end{array}$ & CG & Belalcázar \\
\hline Pósito & CG & Belalcázar \\
\hline Castillo de Gahete & BIC (Monumento) & Belalcázar \\
\hline Convento de Santa Clara de la Columna & BIC (Monumento) & Belalcázar \\
\hline Castillo & BIC (Monumento) & Belmez \\
\hline Castillo del Junquillo & BIC (Monumento) & Belmez \\
\hline Castillo del Viandar en el Hoyo & BIC (Monumento) & Belmez \\
\hline Dolmen Casas de Don Pedro & BIC (Zona Arqueológica) & Belmez \\
\hline Pósito & CG & Belmez \\
\hline Yacimiento Ermita de San Gregorio en Nava Grande & CG & Conquista \\
\hline Las Parras I & CG & Conquista \\
\hline Las Parras II & CG & Conquista \\
\hline Los Prados I & CG & Conquista \\
\hline Los Prados II & CG & Conquista \\
\hline La Ventilla & CG & Conquista \\
\hline Peñascal del Sebo & CG & Conquista \\
\hline Iglesia de Ntra. Sra. de la Asunción & BIC (Monumento) & Dos Torres \\
\hline
\end{tabular}




\begin{tabular}{|c|c|c|}
\hline Centro Histórico de Dos Torres & BIC (Conjunto Histórico) & Dos Torres \\
\hline Pósito & CG & Dos Torres \\
\hline Pósito & CG & Espiel \\
\hline Castillo del Vacar & BIC (Monumento) & Espiel \\
\hline Castillo Cabeza de Vaca & BIC (Monumento) & Espiel \\
\hline Castillo de Sierra del Castillo & BIC (Monumento) & Espiel \\
\hline Ruinas Visigodas del Cerro del Germo & BIC (Monumento) & Espiel \\
\hline Casa Cardona & BIC (Monumento) & Fuente Obejuna \\
\hline Mina de la Loba & CG & Fuente Obejuna \\
\hline Majadaiglesia & BIC (Zona Arqueológica) & El Guijo \\
\hline Ermita de Santa Ana & BIC (Monumento) & Hinojosa del Duque \\
\hline Iglesia de San Juan Bautista & BIC (Monumento) & Hinojosa del Duque \\
\hline Cerro del Castillo del Cohete & BIC (Monumento) & Hinojosa del Duque \\
\hline Castillo & BIC (Monumento) & Hornachuelos \\
\hline Jardín de la Finca Moratalla & BIC (Jardín Histórico) & Hornachuelos \\
\hline Iglesia de Santa María de las Flores & BIC (Monumento) & Hornachuelos \\
\hline Muralla Urbana & BIC (Monumento) & Hornachuelos \\
\hline Finca Moratalla & BIC (Jardín Histórico) & Hornachuelos \\
\hline Antigua Posada & BIC (Lugar de Interés Etnológico) & Hornachuelos \\
\hline Iglesia de San Calixto & CG & Hornachuelos \\
\hline Antiguo Convento de Ntra. Sra. de la Concepción & BIC (Monumento) & Pedroche \\
\hline Ermita de Santa María del Castillo & BIC (Monumento) & Pedroche \\
\hline Iglesia El Salvador & BIC (Monumento) & Pedroche \\
\hline La Jurada & CG & Pedroche \\
\hline El Boquerón & CG & Pedroche \\
\hline La Motilla I & CG & Pedroche \\
\hline La Motilla II & CG & Pedroche \\
\hline La Motilla III & CG & Pedroche \\
\hline El Morteruelo & CG & Pedroche \\
\hline Los Plazares & CG & Pedroche \\
\hline Las Rozuelas & CG & Pedroche \\
\hline La Dehesa & CG & Pedroche \\
\hline Piedra Merendera & CG & Pedroche \\
\hline Haza de Beneficencia & CG & Pedroche \\
\hline Cañada de las Vigas I & CG & Pedroche \\
\hline Cañada de las Vigas II & CG & Pedroche \\
\hline El Saltadero & CG & Pedroche \\
\hline Iglesia de Nuestra Sra. del Rosario & BIC (Monumento) & Peñarroya-Pueblonuevo \\
\hline Abrigo Carmelo & BIC (Monumento) & Peñarroya-Pueblonuevo \\
\hline Cueva de la Osa & BIC (Monumento) & Pozoblanco \\
\hline Pósito & CG & Pozoblanco \\
\hline Castillo de Miramontes & BIC (Monumento) & Santa Eufemia \\
\hline Muralla Urbana & BIC (Monumento) & Santa Eufemia \\
\hline Castillo de Vioque & BIC (Monumento) & Santa Eufemia \\
\hline Muralla Urbana & BIC (Monumento) & Torrecampo \\
\hline Castillo de Almogávar & BIC (Monumento) & Torrecampo \\
\hline La Nava & CG & Torrecampo \\
\hline Haza de las Ánimas I & CG & Torrecampo \\
\hline
\end{tabular}




\begin{tabular}{|l|l|l|}
\hline Haza de las Ánimas II & CG & Torrecampo \\
\hline Cañada del Trillo & CG & Torrecampo \\
\hline Cercado de las Norias & CG & Torrecampo \\
\hline La Cruz Chiquita & CG & Torrecampo \\
\hline Huerta de los Álamos & CG & Torrecampo \\
\hline La Pelicarda & CG & Torrecampo \\
\hline La Torre & CG & Torrecampo \\
\hline Laguna del Ladrillar & CG & Torrecampo \\
\hline Las Coronadas & CG & Torrecampo \\
\hline Las Valquemadas & CG & Torrecampo \\
\hline Castillo de Cinta & BIC (Monumento) & Valsequillo \\
\hline Edificio de la Audiencia & CG & Villanueva de Córdoba \\
\hline Pósito & CG & Villanueva de Córdoba \\
\hline Castillo La Solana & BIC (Monumento) & Villanueva del Rey \\
\hline Castillo Cuzna & BIC (Monumento) & Villanueva del Rey \\
\hline Castillo & BIC (Monumento) & Villanueva del Rey \\
\hline Castillo de Madroñiz & BIC (Monumento) & El Viso \\
\hline
\end{tabular}

BIC: Bien de Interés Cultural; CG: Elemento inscrito en el catálogo general

Fuentes: Instituto Andaluz de Patrimonio Histórico. Consejería de Medio Ambiente y Ordenación del Territorio (Junta de Andalucía). Trabajo de campo. Elaboración propia

Figura 4. Localización del patrimonio territorial institucionalizado en Los Pedroches-Valle del Guadiato

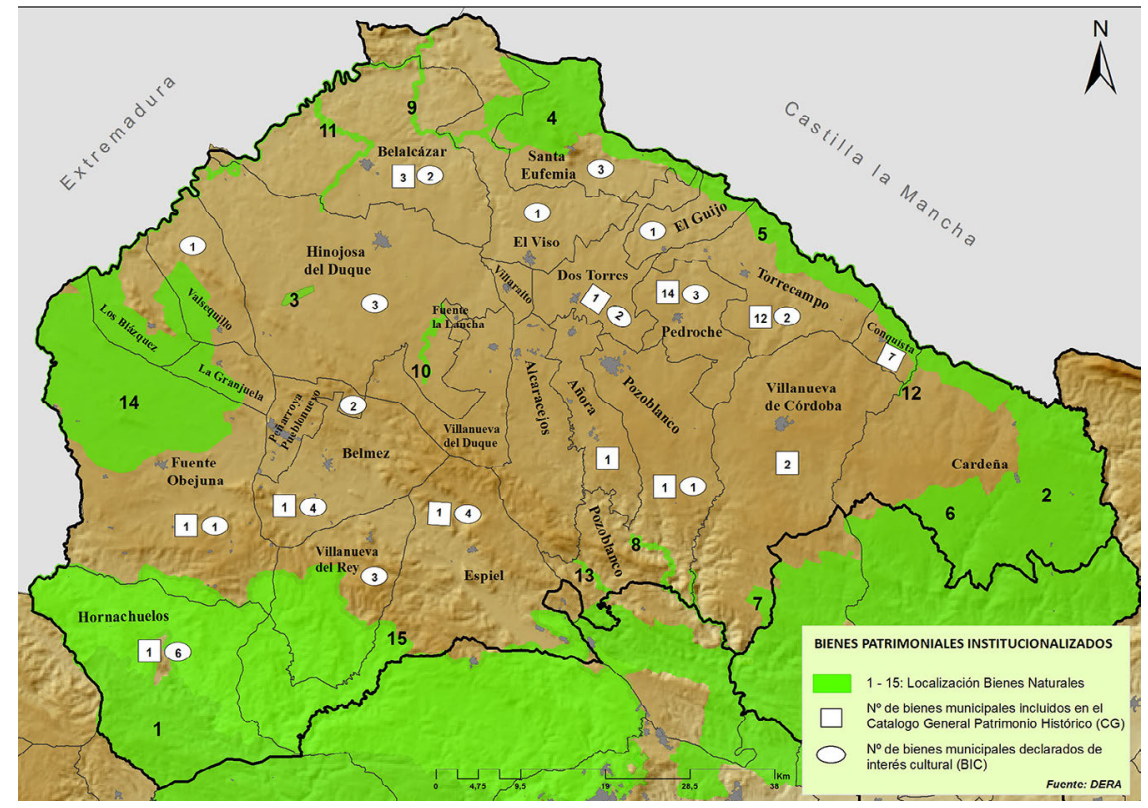

Fuente: Datos Espaciales de Referencia en Andalucía (DERA) y trabajo de campo. Elaboración propia

\subsection{El tercer escollo: la heterogeneidad normativa y la escasa imbricación de los instrumentos básicos de planificación en lo concerniente al patrimonio}

A pesar del consenso teórico en torno al sinsentido de la división tradicional entre patrimonio natural y patrimonio cultural, lo cierto es que, en el plano normativo, la misma sigue siendo una realidad revestida aún de considerable fuerza. No sólo existe una legislación estatal básica y diferenciada para cada "tipo" de patrimonio, sino que, además, el conjunto de los bienes reconocidos por el POTA como integrantes 
del Sistema de Patrimonio Territorial de Andalucía, se encuentra regulado por un elenco igualmente complejo de normas e instrumentos internacionales, estatales y autonómicos, de una casi imposible armonización -y muy especialmente en lo relacionado con el patrimonio natural-; no obstante, lo anterior se atenúa considerablemente si sólo nos atenemos a los bienes de inconfundible sello institucional, que han sido tratados en el epígrafe anterior.

Por otra parte, en lo concerniente a los grandes instrumentos de planificación vigentes, es evidente la separación entre planificación territorial y planificación sectorial. Por ejemplo, el establecimiento de las Unidades Territoriales recogidas en el POTA -referente esencial para la gestión integrada del patrimoniose llevó a cabo sin atender siquiera a los límites de las grandes piezas patrimoniales ya institucionalizadas: como resultado, algunos de los más importantes espacios protegidos presentes en el ámbito analizado (Parque Natural de la Sierra de Hornachuelos, Parque Natural de la Sierra de Cardeña-Montoro, Reserva de la Biosfera Dehesas de Sierra Morena) se encuentran compartimentados entre varias Unidades Territoriales limítrofes, con las dificultades de todo tipo que ello plantea. De otro lado, los instrumentos básicos de la planificación ambiental (Planes de Ordenación de los Recursos Naturales y Planes Rectores de Uso y Gestión de los dos parques naturales aludidos), aprobados en 2003 pero revisados y prorrogados en 2011, no hacen referencia específica a las nuevas directrices en materia de patrimonio territorial establecidas en el POTA, como tampoco a la conveniencia de avanzar en pos de una gestión armónica de los elementos patrimoniales presentes en sus respectivos ámbitos de actuación. En definitiva, son muchos los desajustes en la línea citada, pero la mayor parte de ellos podrían haberse evitado mediante la elaboración de las Estrategias de planificación y gestión integrada del Patrimonio Territorial ${ }^{6}$, instituidas en el Plan de Ordenación del Territorio con carácter de norma, y destinadas a "establecer un marco de actuación común para cada territorio en el que se integren tanto las políticas patrimoniales (ecológicas, culturales y paisajísticas) como las políticas urbanísticas, de ordenación del territorio y de desarrollo económico, particularmente aquellas relacionadas con la promoción turística vinculada a la puesta en valor de los bienes patrimoniales" (POTA, 2006, 112).

\section{CONCLUSIONES}

Desde los pasados años ochenta han proliferado extraordinariamente las intervenciones en materia de protección patrimonial. Las numerosas amenazas y riesgos que, en el actual contexto globalizador, se ciernen sobre el territorio, el mayor reconocimiento social de los bienes patrimoniales y la concepción cada vez más laxa y abierta de los que deben considerarse como tales son -junto a su reconocido potencial como elementos dinamizadores de las economías locales- algunas de las razones explicativas de tal expansión protectora.

En términos territoriales la magnitud de los bienes y espacios catalogados, y la evidente relación entre los mismos, obliga a una reinterpretación del bien patrimonial. Éste deja de valorarse como elemento aislado y dotado de una protección específica -de índole museística-, para ser entendido como pieza destacada pero imbricada y gestionada en su contexto territorial. Y, en estrecha vinculación ello, se insiste en la necesidad de superar la tradicional separación entre patrimonio natural y patrimonio cultural, aunque en el plano jurídico tal pretensión está lejos de alcanzarse.

Pues bien, desde los anteriores planteamientos, algunas iniciativas institucionales -con distinto origen y alcance- están suponiendo un claro avance en el entendimiento integrado del patrimonio territorial. Entre las mismas, resulta sugerente la selección y protección de determinados paisajes, al considerarse que son ilustrativos de los estrechos vínculos existentes entre naturaleza y cultura; sin duda, las figuras de Paisaje Protegido y Paisaje Cultural -auspiciadas por UICN y UNESCO, respectivamente-, son dos logros destacados en la citada línea, y ambas gozan ya de una notoria traslación al territorio español. No obstante, se han impulsado herramientas de mayor interés y alcance que, más allá de una intención ejemplificadora, aspiran a lograr la gestión integrada de los bienes patrimoniales radicados en un ámbito determinado. Una de ellas es el Sistema de Patrimonio Territorial de Andalucía (SPTA), que ha sido analizada en su condición de iniciativa pionera en la materia, y al respecto de la cual cabe hacer las siguientes consideraciones generales:

6 El POTA $(2006,112)$ encomienda su redacción a las entonces Consejerías de Agricultura y Pesca, Cultura, Medio Ambiente, Turismo, Comercio y Deportes y Obras Públicas y Transportes, a través de un Programa Coordinado. 
$1^{\text {a }}$ En el contexto actual, resultan muy convenientes propuestas de esta índole, dirigidas a establecer mecanismos específicos para el tratamiento conjunto y coordinado de los bienes patrimoniales de carácter territorial. En el caso andaluz, además, esta iniciativa se encuentra plenamente justificada por el elevado número de elementos que disponen ya de algún grado de protección, la heterogeneidad jurídica que los caracteriza y la insuficiente imbricación de los mismos.

$2^{a}$ No obstante, una evaluación detallada de los instrumentos y directrices del SPTA, pone de manifiesto las considerables dificultades que encuentra su aplicación práctica. Unas limitaciones que llegan incluso a comprometer su viabilidad futura y, en consecuencia, la credibilidad del propio POTA; entre las más significativas han de citarse la incapacidad para establecer una división territorial coherente sobre la que asentar la pretendida gestión integrada de los elementos patrimoniales, la propia indefinición de las piezas que han de conformar el catálogo de bienes del patrimonio territorial y, por último, la escasa armonización existente entre planificación territorial y sectorial.

Finalmente, el estudio de caso llevado a cabo en la Unidad Territorial Los Pedroches-Valle del Guadiato ha permitido constatar sobre el terreno la existencia de los problemas estructurales antes referidos, a la vez que mostrar la debilidad de la intervención institucional y la absoluta desvinculación existente entre los principales bienes patrimoniales de índole cultural y natural allí radicados. El norte cordobés, por otra parte, se perfila como un buen ejemplo de los que podrían denominarse territorios marginales en el contexto de los criterios imperantes en la patrimonialización institucional de las últimas décadas. Su imagen de marca, muy potente en términos agrarios y funcionales, ha contribuido a alejarlo aún más de aquellos otros ámbitos que, en virtud de su menor dinamismo y su mayor apariencia de naturalidad, han sido considerados prioritarios a los efectos de protección y catalogación, y de ahí el escaso patrimonio territorial que se le ha reconocido. Baste citar, como muestra de lo afirmado, que Los Pedroches, donde se desarrolla una de las dehesas mejor constituidas de Andalucía -sino la mejor-, han quedado fuera incomprensiblemente de la extensísima Reserva de la Biosfera Dehesas de Sierra Morena (424.000 ha), declarada en 2002 conforme a los criterios antedichos.

\section{RECURSOS ELECTRÓNICOS}

CASTILLO, J. (2007): "El futuro del patrimonio histórico: la patrimonialización del hombre". Revista de Patrimonio Histórico (Universidad de Granada), nº 1 (edición digital).

EUROPARC (2012): Anuario 2011 del estado de las áreas protegidas en España. Madrid, Fundación Fernando González Bernáldez, 187 p.

FERIA, J.M. (2010): "Patrimonio territorial y desarrollo sostenible: un estudio comparativo en Iberoamérica y España". Estudios Geográficos, n 268, p. 129-159.

FERNÁNDEZ, S. et al. (2010) Paisaje y patrimonio cultural en Andalucía. Tiempo, Usos e Imágenes. Sevilla, Instituto Andaluz de Patrimonio Histórico (Junta de Andalucía).

FLORIDO, G. (2013): "El patrimonio territorial en el plan de ordenación del territorio de Andalucía: indefiniciones y dificultades para un conocimiento preciso". Boletín de la Asociación de Geógrafos Españoles, $n^{\circ}$ 63, p. 173-202.

GÓMEZ, J. (2013): "Del patrimonio paisaje a los paisajes patrimonio". Documents d'Anàlisi Geogràfica, vol. 59.1, pp. 5-20.

GRUPO DE DESARROLLO RURAL Y URBANO (GDRU) (2007): "Recursos patrimoniales y organización territorial: el caso de Andalucía". Ciudad y Territorio. Estudios Territoriales, XL (156), pp. 297-311.

MATA, R. (2010): "La dimensión patrimonial del paisaje. Una mirada desde los espacios rurales". En Maderuelo, J. (Dir.): Paisaje y patrimonio. Madrid, CDAN-Abada Editores, pp. 31-73.

MULERO, A. (2001): "Sierra Morena como espacio protegido. Del olvido tradicional al interés reciente". Investigaciones geográficas, $\mathrm{n}^{\circ} 25$, pp. 20-40.

MULERO, A., GARZON, R. y NARANJO, J. (2011): "The system for territorial heritage and its significance in the context of new procedures and rules for territorial management in Andalusia (Spain)". En Actas de la Conferencia Geográfica Regional de la Unión Geográfica Internacional (UGI). Doc. no publicado. Santiago de Chile, 2011.

MULERO, A. (2013): "Significado y tratamiento del paisaje en las políticas de protección de espacios naturales en España". Boletín de la Asociación de Geógrafos Españoles, nº 62, pp. 129-145.

OJEDA, J.F. (2005): "Percepciones identitarias y creativas de los paisajes mariánicos". Scripta Nova: Revista Electrónica de geografía y ciencias sociales, nº 9, 2005, pp. 181-204. 
ORTEGA, J. (1998): "El patrimonio territorial: el territorio como recurso cultural y económico". Ciudades, $n^{\circ}$ 4. Revista del Instituto de Urbanística de la Universidad de Valladolid, pp. 33-48.

PHILLIPS, A. (ed.) (2002) Management Guidelines for IUNC Category V Protected Areas/Protected Landscapes/Seascapes. Cambridge (UK), IUCN, 122 p.

PLAN DE ORDENACIÓN DEL TERRITORIO DE ANDALUCÍA (POTA). Boletín Oficial de la Junta de Andalucía $\mathrm{n}^{\circ}$ 136, de 17 de julio de 2006. Disponible en http://www.juntadeandalucia.es/medioambiente/ site/portalweb

PRATS, LL. (2004): Antropología y patrimonio. Barcelona, Ariel, $2^{\mathrm{a}}$ Ed.

RODRIGO, J.M. et al. (2012): "Registro de paisajes de interés cultural de Andalucía. Criterios y metodología". Revista ph. Instituto Andaluz de Patrimonio Histórico, no 81, p. 64-75.

RÖSSLER, M. (2002): "Los paisajes culturales y la Convención del Patrimonio Mundial Cultural y Natural: resultados de reuniones temáticas previas", en MÚJICA BARREDA, E. (ed.), Paisajes culturales en los Andes: memoria narrativa, casos de estudio, conclusiones y recomendaciones de la Reunión de expertos, Arequipa y Chivay, Perú, mayo de 1998, Lima, UNESCO, pp. 49-57.

SILVA, R. (2009): "Agricultura, paisaje y patrimonio territorial. Los paisajes de la agricultura vistos como patrimonio". Boletín de la AGE, n 49, pp. 309-334.

TROITIÑO, M.A. (2011): "Territorio, patrimonio y paisaje: desafíos de una ordenación y gestión inteligentes". CYTET, n 169-170, pp. 561-569.

ZOIDO, F. (2011): "Ordenación del Territorio en Andalucía. Reflexión personal". Cuadernos Geográficos, $n^{\circ} 47$, pp. 189-211. 